\title{
An Unusual Case of Acute Dyspnoea: Acute Intrathoracic Gastric Volvulus with Probable Tension Gastrothorax
}

\author{
Jean Bosco Masabarakiza ${ }^{1}$, Lingna Zhu ${ }^{1}$, Yilmaz Gorur ${ }^{2}$, Benoît Cardos ${ }^{3}$, Noel Lorenzo-Villalba ${ }^{4}$ Deeba Ali $^{5}$ \\ ${ }^{1}$ Service des Urgences, CHU Sart-Tilman, Liège, Belgium \\ ${ }^{2}$ Service de Radiologie, Clinique André Renard, Herstal, Belgium \\ ${ }^{3}$ Service des Urgences, Clinique André Renard, Herstal, Belgium \\ ${ }^{4}$ Service de Médecine Interne, Diabète et Maladies Métaboliques, Hôpitaux Universitaires de Strasbourg, France \\ ${ }^{5}$ Service des Urgences, CHC MontLégia, Liège, Belgium
}

\begin{abstract}
Received: 05/08/2021
Accepted: 13/08/2021

Published: 06/10/2021 volvulus with probable tension gastrothorax. EJCRIM 2021;8: doi:10.12890/2021_002818.

Conflicts of Interests: The authors declare there are no competing interests.

Acknowledgements: The authors thank Lena Andriollo for her editing services and support.

This article is licensed under a Commons Attribution Non-Commercial 4.0 License
\end{abstract}

How to cite this article: Masabarakiza JB, Zhu L, Gorur Y, Cardos B, Lorenzo-Villaba N, Ali D. An unusual case of acute dysponea: acute intrathoracic gastric

\section{ABSTRACT}

Acute gastric volvulus is a rare complication affecting $4 \%$ of hiatal hernias. It is described as an abnormal rotation of the stomach around a transverse or longitudinal axis and can be intra-abdominal or intrathoracic. Intrathoracic gastric volvulus can lead to tension gastrothorax, which is defined as the presence of a massive gastric distension in the chest cavity causing a mediastinal shift with haemodynamic instability and a risk of cardiorespiratory arrest. We report a case of intrathoracic gastric volvulus with probable tension gastrothorax. Early diagnosis of gastrothorax with computed tomography and immediate insertion of a nasogastric tube resulted in rapid clinical improvement. Surgical intervention is the treatment of choice.

\section{LEARNING POINTS}

- Tension gastrothorax should be suspected in the presence of abdominal and chest pain, vomiting and haemodynamic instability.

- $\quad$ Prompt diagnosis and treatment are necessary to reduce morbidity and mortality.

\section{KEYWORDS}

Volvulus gastric, hiatal hernia, gastrothorax

\section{INTRODUCTION}

Gastric volvulus was first described by Berti in 1886. It is defined as torsion of the stomach around one of its axes ${ }^{[1]}$. It is a rare condition complicating $4 \%$ of hernias and requires urgent surgical intervention ${ }^{[2]}$. The clinical symptomatology is mostly non-specific, which often delays the diagnosis.

It is usually an intra-abdominal condition, but there is a rare intrathoracic variant related to passage of the stomach into the mediastinum through an abnormal diaphragmatic opening (congenital or acquired).

We report a case of intrathoracic gastric volvulus with probable development of a tension gastrothorax in a patient with hiatal hernia. 


\section{CASE DESCRIPTION}

A 55-year-old woman was admitted to the emergency department with epigastric pain associated with vomiting and dyspnoea for more than 24 hours. Her past medical history was relevant for hiatal hernia, pulmonary embolism and arterial hypertension.

On admission, she was hypotensive (systolic blood pressure $80 \mathrm{mmHg}$ ), tachycardic (140 beats/min), respiratory rate was 25/min and she was saturating $85 \%$ on room air. She had a diminished level of consciousness but rapid arousal to verbal stimuli. The abdomen was soft and slightly painful on palpation in the epigastric region. Several episodes of vomiting were noted during clinical examination.

In this clinical setting, a nasogastric tube was inserted leading to clinical improvement (systolic blood pressure increased to $119 \mathrm{mmHg}$ and oxygen saturation to 90\%). The nasogastric tube drained $3200 \mathrm{ml}$ of brownish fluid.

Laboratory investigations showed anaemia (haemoglobin $8.7 \mathrm{~g} / \mathrm{dl}$ ), acute renal failure (creatinine clearance $22 \mathrm{ml} / \mathrm{min}$, glomerular filtration rate), hyperleukocytosis $\left(22,000 / \mathrm{mm}^{3}\right)$, and increased C-reactive protein $(63 \mathrm{mg} / \mathrm{l})$ and lactates $(5.4 \mathrm{mmol} / \mathrm{l})$. The thoracoabdominal scan revealed a large hiatal hernia, an intrathoracic gastric volvulus with significant gastric distension, fluid stasis in the oesophagus and gastric wall pneumatosis. (Figs. 1-3). These clinical, laboratory and imaging results were in favour of an intrathoracic gastric volvulus complicated by probable tension gastrothorax.

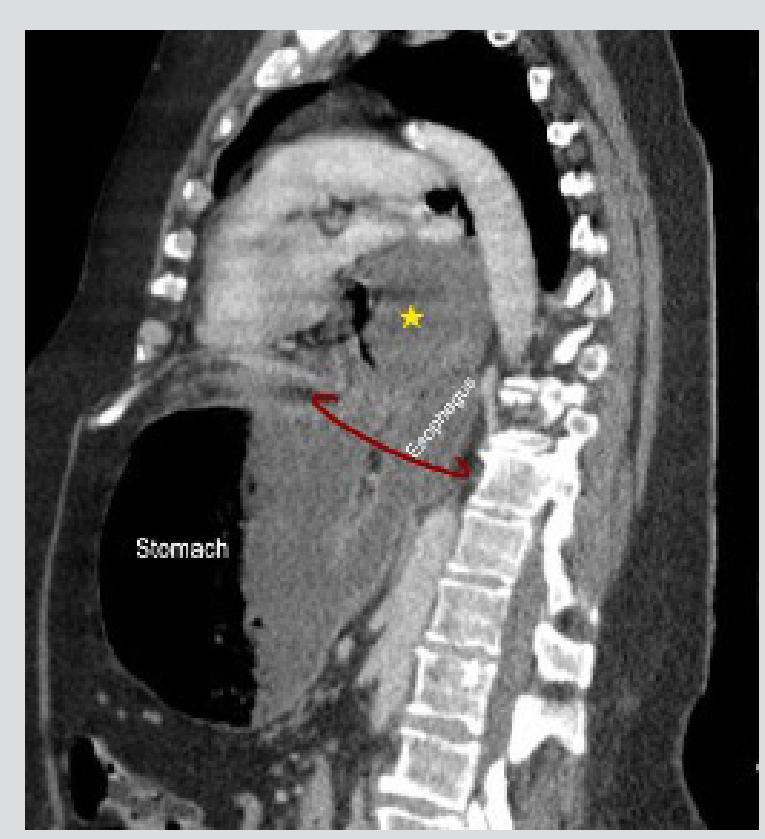

Figure 1. CT scan (portal phase in the sagittal view) showing a hiatal hernia (red line) with intussusception of the intrathoracic part (star)

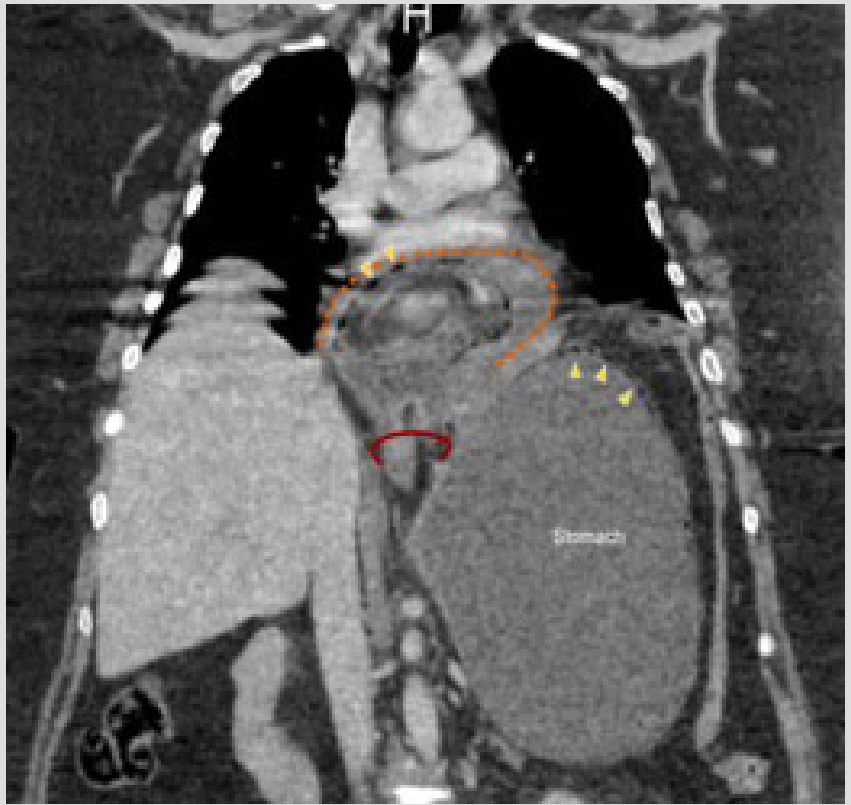

Figure 2. CT scan (portal phase in the coronal view) showing the hiatal hernia with intussusception of the thoracic part of the stomach (delimited by the dotted line). Gastric pneumatosis in the thoracic and abdominal parts of the stomach is indicated by arrowheads

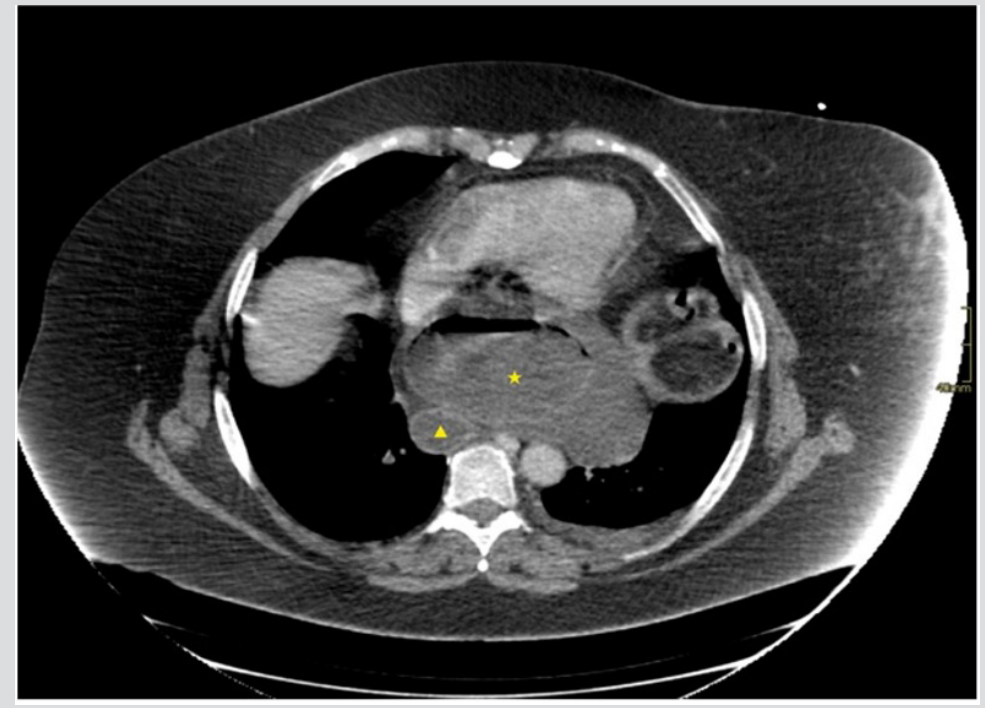

Figure 3. CT scan (transverse axial section) with intravenous contrast. The star indicates the hiatal hernia caused by twisting of the stomach complicated by gastric invagination. The oesophagus, marked with a yellow triangle, is adjacent to the intrathoracic stomach 
The patient's general condition progressively deteriorated, requiring respiratory assistance with mechanical ventilation and she was transferred to the intensive care unit. Upper endoscopy revealed ischaemic lesions in the gastric mucosa without necrosis. Surgical intervention was considered. Laparoscopic surgery confirmed the diagnosis of gastric volvulus within a large hiatal hernia. Reduction of the volvulus with reintegration of the stomach into the abdominal cavity and a gastropexy were performed.

The procedure was conducted without any further complications. The patient was extubated 3 days after surgical intervention and she was transferred to a regular hospital ward 2 weeks after admission to intensive care.

\section{DISCUSSION}

Gastric volvulus is a rare and potentially serious condition depending on its complications. These may include gastrointestinal haemorrhage if there is gastric mucosal damage, peritonitis if gastric perforation, or tension gastrothorax. The incidence of gastric volvulus is highest after the age of 50 years, although $10-20 \%$ of cases occur in children under 1 year of age ${ }^{[3]}$. Gastric volvulus may be either idiopathic or secondary to diaphragmatic or intraperitoneal abnormalities.

Gastric volvulus usually presents in the abdomen, but it can sometimes be found in the intrathoracic cavity as a result of the passage of the stomach through a congenital, iatrogenic or traumatic diaphragmatic anomaly or the existence of a hiatal hernia.

The clinical presentation of gastric volvulus is not very specific and varies according to its acute or chronic character, the degree of gastric obstruction, and the location. It may present as an acute abdomen or as respiratory complaints (dyspnoea, hiccups, chest pain). The classic Borchardt triad is present in approximately $70 \%$ of cases and includes epigastric pain and/or sudden chest pain, abdominal distension with ineffective vomiting efforts and difficulty or impossibility of placing a nasogastric tube. This clinical picture is highly suggestive of acute gastric volvulus ${ }^{[4]}$.

In its thoracic location, acute gastric volvulus may present as a tension gastrothorax, a life-threatening complication. Tension gastrothorax develops as the stomach fills with air, fluid or food within the thoracic cavity through a one-way valve mechanism created by abnormal angulation of the gastroesophageal junction and obstruction of the gastric outlet at the abnormal opening of the diaphragm [5]. Mediastinal deviation may cause large vessel obstruction leading to obstructive shock with a risk of cardiorespiratory arrest.

In the case presented, the hypothesis of tension gastrothorax was plausible because of the initial clinical findings, the haemodynamic instability on admission, and the clear improvement in clinical condition after nasogastric tube insertion. However, further investigations are essential to confirm the diagnosis. Intrathoracic gastric volvulus is a possibility if an abdominal organ is found in the mediastinum on chest radiography. Observation of oesophageal-duodenal transit allows determination of the reducibility of the gastric volvulus, its intraabdominal or intrathoracic position, and antropyloric evacuation of the contrast medium, which is often difficult.

Upper endoscopy permits determination of the cause (such as a hiatal hernia) and evaluation of the gastric mucosa. This procedure allows endoscopic reduction of the gastric volvulus and guided percutaneous gastropexy ${ }^{[6]}$.

Thoracoabdominal CT is the gold standard to confirm the diagnosis. It allows the type and aetiology of the volvulus to be determined, as well as evaluation of the cardiopulmonary consequences of the intrathoracic migration of the stomach. The use of contrast allows the digestive wall to be examined, and vascular damage and areas of parietal necrosis to be determined, thus indicating if urgent surgery is necessary ${ }^{[7]}$. The management of intrathoracic gastric volvulus in the emergency department consists of decompressing the stomach to reduce tension. The first decompression attempt should be performed via nasogastric tube placement with possible use of gastrointestinal endoscopy guidance to improve the chances of success ${ }^{[8,9]}$.

In cases where this procedure fails, gastric dilatation and tensioning may lead to obstructive shock due to mediastinal bypass and even cardiorespiratory arrest. Urgent transthoracic needle decompression of the stomach is essential to stabilize the patient before surgical management is initiated ${ }^{[8,9]}$.

Surgery is the treatment of choice for gastric volvulus. The surgical procedure consists of devolvulation with reduction of the stomach into the abdominal cavity and gastropexy or repair of the diaphragmatic defect ${ }^{[10]}$.

\section{CONCLUSION}

Intrathoracic gastric volvulus is a rare complication of hiatal hernia that can be fatal if tension gastrothorax develops. CT scanning allows confirmation of the diagnosis, assessment of thoracic lesions, and evaluation of the viability of the gastric wall. The treatment of choice remains surgical, although gastric decompression with a nasogastric tube is the first-line treatment in the emergency room. 


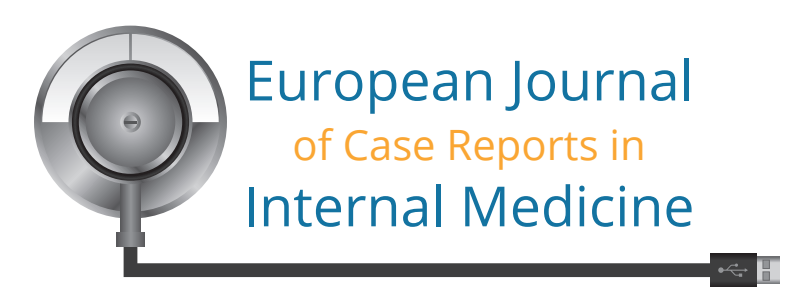

\section{REFERENCES}

1. Hillemand P, Bernard HJ, Villard J. À propos des volvulus gastriques. Sem Hop Paris 1955;31:2890-2899.

2. Marini L, Azarian R, Gagadoux F, Brion N, Petitpretz P. Gastric volvulus associated with Zenker's diverticulum: a rare cause of recurrent pneumopathy. Rev Mal Respir 2000;17:121-123.

3. Ferreira CR, Maximiano LF, Santos VML, Martines JAS. Intrathoracic gastric volvulus: an autopsy case report. Autops Case Rep 2013;3(2):21-29.

4. Rashid A, Thangarajah T, Mulvey D, Larvin M, Iftikhar AY. A review article on gastric volvulus: a challenge to diagnosis and management. Int J Surg 2010;8:18-24.

5. Horst M, Sacher P, Molz G, Willi UV, Meuli M. Tension gastrothorax. J Pediatr Surg 2005;40:1500-1504.

6. Franco A, Vaughan KG, Vukcevic Z, Thomas S, Mazariegos GV. Gastric volvulus as a complication of liver transplant. Pediatr Radiol 2005;35:327-329.

7. Grignon B, Sebbag H, Reibel N, Zhu X, Grosbidier G, Rolond J. Diagnostic tomodensitometrique d'un volvulus gastrique idiopathique aigue. J Radiol 2004;85:1070-1073.

8. Naess PA, Wiborg J, Kjellevold K, Gaarder C. Tension gastrothorax: acute life-threatening manifestation of late onset congenital diaphragmatic hernia (CDH) in children. Scand J Trauma Resusc Emerg Med 2015;23:49-50.

9. Bamgbade OA. Management of tension gastrothorax. Resuscitation 2006;70:293-294.

10. Bedioui H, Bensafta Z. Volvulus gastrique: diagnostic et prise en charge thérapeutique. Presse Med 2008;37:67-76. 